Kyle Burton

IU School of Medicine

\title{
Determining the Mechanism of Propofol-induced Neurotoxicity
}

\author{
Kyle Burton ${ }^{1}$, Scott Canfield, $\mathrm{PhD}^{1}$ \\ ${ }^{1}$ Indiana University School of Medicine, Terre Haute
}

Background and Hypothesis: Propofol is an IV anesthetic agent with many clinical applications, including general anesthesia, sedation, delirium, and palliative care. Despite its versatility and effectiveness, Propofol has been shown to have detrimental effects on the vascular interface between the brain and circulation. The Blood-Brain Barrier (BBB) acts as a selective interface that serves to protect the brain from its cerebrovascular network. BBB dysfunction can have lethal complications such as cerebral edema and stroke. Matrix Metalloproteinases (MMP's), specifically MMP-2 and MMP-9 have been linked to BBB breakdown. Using human induced pluripotent stem cell (IPSC) derived brain microvascular endothelial cells (BMECs), we evaluated the impact of inhibiting MMP-2 on restoring BBB integrity following exposure to Propofol with the intent to reveal the mechanism by which Propofol disrupts the BBB.

We hypothesized that inhibiting MMP-2 would lead to phenotype recovery after exposure to Propofol.

Methods: IPSC-differentiated BMECs were treated with MMP inhibitors at varying time points and concentrations relative to exposure to Propofol for 3 hours. Trans-endothelial electrical resistance (TEER) and sodium fluorescein permeability was used to assess BBB structural integrity. A MTT assay was conducted to assess cell viability.

Results: Inhibiting MMP-2 did not result in in recovery of BBB integrity following exposure to Propofol as no significant differences were observed in TEER and sodium fluorescein permeability between the Propofol control and Propofol + MMP-2 inhibitor groups.

Conclusions \& Future Directions: Propofol-induced disruption of BBB integrity does not appear to be through MMP-2 activity. High concentrations of MMP-inhibitor compounds result in increased disruption of BBB tightness and permeability. It is plausible to suspect that Propofol may act through other MMP's to facilitate BBB break-down, thus future studies should investigate the effects of the other selective MMP inhibitors in their ability to achieve phenotype recovery following exposure to Propofol. 